\title{
Antropología e interculturalidad en la primera infancia: desde el cuidado de enfermería fundamentado en ritos de paso en una comunidad indigena pume
}

\author{
Anthropology and interculturality in first childhood: from the nursing care based on \\ passage rites in an indigenous pume community
}

\section{Antropologia e interculturalidade na primeira infância: do cuidado de enfermagem com base em ritos de passagem em uma comunidade indígena}

\section{Zaida Colmenares Robles, ${ }^{1}$ Delia Moya Plata $^{2}$, Isoled Herrera ${ }^{3}$}

${ }^{1}$ Profesora Asociado a Dedicación Exclusiva. Doctor en Ciencias Sociales. Mención Salud y Sociedad Universidad de Carabobo. Valencia. Venezuela. Profesora Asociado a Dedicación Exclusiva Doctor en Ciencias Sociales. Mención Salud y Sociedad. Universidad de Carabobo. Valencia. Venezuela.

${ }^{2}$ Profesora en la Universidad de Santander UDES, Facultad Ciencias de la Salud, Programa de Enfermería. Grupo de Investigación Everest. Bucaramanga (Colombia). Enfermera Magíster en Salud Familiar. Enfermera Especialista en Salud Ocupacional. Enfermera Especialista en Docencia Universitaria. Profesora Titular de dedicación exclusiva. Coordinadora Grupo de Cuidado del Programa de Enfermería UDES.

${ }^{3}$ Profesora en la Universidad Península de Santa Elena. Facultad Ciencias de la Salud. UPSE. Carrera de Enfermería. Especialista en Docencia Universitaria. Doctora en Ciencias de la Educación. Ecuador

Cómo citar este artículo en edición digital: Colmenares Robles, Z., Moya Plata, D., \& Herrera. I. (2020). Antropología e interculturalidad en la primera infancia: desde el cuidado de enfermería fundamentado en ritos de paso en una comunidad indígena pume. Cultura de los Cuidados (Edición digital), 24 (57) Recuperado de http://dx.doi.org/10.14198/cuid.2020.57.19

Correspondencia: Residencias Bella vista country club en Av. Bolívar vieja de Naguanagua, ValenciaVenezuela Correo electrónico: zcolmena.uc.edu.ve-deliamoyaplata@hotmail.com

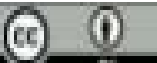

\begin{abstract}
Introduction: Nursing practice should promote the maintenance of wellbeing and life, through respect for culture against disease, through models of care in the evidence-based pluricultural context.
\end{abstract}

The objective of this study was to reveal the rites of early childhood Pumé, and its impact on cultural reproduction and health care found such as protective collars, the placement of clinical names and the predisposition of the child: writing and painting skills attributed to maturity during its growth. Methodology: We frame the look 
in a focused ethnography exploring interculturality, rites of steps. The group of social actors subject to study was made up of seven key informants of the Pumé Ethnicity, with more than 5 years in the Community studied. Conclusions: Cultural care in Early Childhood will include activities aimed at preserving or maintaining, adapting or negotiating, reorienting or restructuring the care granted to children. That is why in the multicultural context in which we live, it is important to incorporate beliefs and values of people and thus provide effective, satisfactory and coherent nursing care with their culture and with evidence-based nursing practice

Keywords: Nursing, rites of passage, anthropology, interculturality

\section{RESUMEN}

Introducción: $\mathrm{La}$ práctica de enfermería, tendría que promover el mantenimiento del bienestar y la vida, mediante el respeto a la cultura frente a la enfermedad, a través de modelos de cuidados en el contexto pluricultural basado en la evidencia. El objetivo de este estudio fue Desvelar los ritos de la primera infancia Pumé, y su incidencia en la reproducción cultural y los cuidados de salud hallados como son los collares protectivos, la colocación de nombres clánicos y la predisposición del niño: habilidades para escribir y pintar atribuido a la madurez durante su crecimiento. Metodología: Enmarcamos la mirada en una etnografía focalizada explorando la interculturalidad, ritos de pasos. El grupo de actoras sociales sujeto de estudio, estuvo conformado por siete informantes claves de la Etnia Pumé, con más de 5 años en la Comunidad estudiada. Conclusiones: Los cuidados culturales en la Primera infancia, comprenderán actividades orientadas a preservar o mantener, adaptar o negociar, reorientar o reestructurar los cuidados otorgados a las niños Es por ello que en el contexto multicultural en el que vivimos, toma importancia incorporar las creencias y valores de las personas y de esta forma proporcionar cuidados de enfermería efectivos, satisfactorios y coherentes con su cultura y con la práctica de enfermería basada en evidencia

Palabras claves: Enfermería, ritos de paso, antropología, interculturalidad.

\section{RESUMO}

Introdução: A prática de enfermagem deve promover a manutenção do bem-estar e da vida, através do respeito à cultura contra a doença, através de modelos de cuidado no contex to pluricultural baseado em evidências. O objetivo deste estudo foi revelar os ritos da infância de Pumé e seu impacto na reprodução cultural e nos cuidados de saúde encontrados como colares protetores, colocação de nomes clínicos e predisposição da criança: habilidades de escrita e pintura atribuídos à maturidade durante o seu crescimento. Metodologia: Enquadramos o visual em uma etnografia focada, explorando a interculturalidade, ritos de etapas. $\mathrm{O}$ grupo de atores sociais sujeitos a estudo era composto por sete informanteschave da etnia Pumé, com mais de cinco anos na Comunidade estudada. Conclusões: $\mathrm{O}$ cuidado cultural na primeira infância incluirá atividades que visam preservar ou manter, adaptar ou negociar, reorientar ou reestruturar o cuidado concedido às crianças, por isso, no contexto multicultural em que vivemos, é importante incorporar crenças e valores das pessoas e, assim, prestar assistência de enfermagem eficaz, satisfatória e coerente com sua cultura e com a prática de enfermagem baseada em evidências

Palavras-chave: Enfermagem, ritos de passagem, antropologia, interculturalidade.

\section{INTRODUCCION}

Entramado de la $\quad$ construcción
sociocultural de la $\begin{gathered}\text { Enfermería } \\ \text { transcultural en la primera infancia }\end{gathered}$


Revista científica de la Asociación de Historia y Antropología de los Cuidados (Universidad de Alicante)

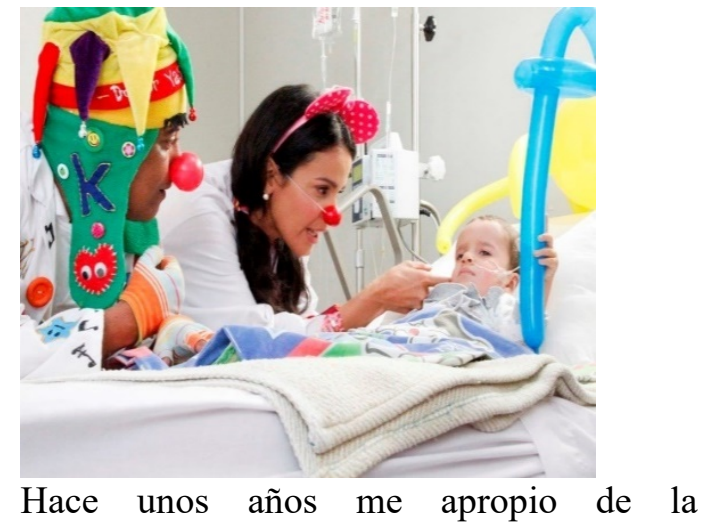

antropología, hacia el cuidado de enfermería, y comienzo a hacer estudios en el área de experticia, que son los niños, sucede en Apure, tiempo de invierno, era un agosto, escenario anual de trabajo voluntario con las etnias indígenas del país. Donde acudo por seis años consecutivos, esta vez fue el estado Apure, en ese momento se necesitaba mi trabajo voluntario dirigido a la salud integral de las etnias Indígenas establecidas en Venezuela. Hago simbiosis en la enfermería positivista, con enfoque biologicista y me traslado al área de los principios culturales en la primera infancia. La enfermería transcultural fundamenta sus raíces en lo citado por la Dra. Madeleine Leininger, presagió que con el tiempo esta área se convertiría en un nuevo dominio del ejercicio de la enfermería, que se reflejará en una práctica profesional diferente, conceptualizado en principios culturales y pensados específicamente para la orientación de los cuidados de la enfermería a los individuos, las familias los grupos y las instituciones. Afirmaba que la enfermería transcultural se ha de convertir en una de las áreas más importantes, significativas $\mathrm{y}$ prometedoras del estudio formal, la investigación y la práctica, debido al mundo multicultural. Aseguraba que, para que enfermería tenga importancia y significado frente al cuidado de enfermería en los niños y otros profesionales de este campo en todo el mundo, es imperativo orientar de conocimientos y competencias en enfermería transcultural, capaces de modificar las acciones y las decisiones para obtener resultados positivos y eficaces.

Establecía la diferencia entre enfermería transcultural e intercultural, en donde la primera se refiere a los profesionales de enfermería preparados y comprometidos en lograr un conocimiento y un procedimiento práctico de actuación de la enfermería transcultural. La enfermería intercultural la constituyen los profesionales que recurren a los conceptos antropológicos médicos o aplicados, sin implicar en el desarrollo de teorías o prácticas fundadas en la investigación, dentro del campo de la enfermería transcultural, otra diferencia es que la enfermería transcultural es la que desarrollan los profesionales que trabajan con dos culturas.

Para proporcionar cuidados culturalmente adecuado emerge la investigación como estrategias creativas que modifiquen el modo de vivir de las diferentes culturas. El propósito de la enfermería transcultural en la infancia, es la prolongación de nuevas formas de ayudar a las personas de diferentes culturas, siendo su objetivo proporcionar cuidados 
culturalmente apropiados de manera que no experimenten contextos negativas como la imposición cultural y el etnocentrismo, concepciones que suelen llevar a choques y conflictos culturales entre clientes $\mathrm{y}$ enfermeras.

De lo anterior, se considera que existe la necesidad de apropiarse más a la realidad social mediante corrientes de pensamientos de aspectos ideológicosculturales, brindando a la práctica de enfermería, herramientas para imaginar e intervenir basado en los postulados de la corriente de los cuidados culturales, que plantea "cultura, creencias de salud o enfermedad, valores y prácticas de las personas, para ayudarlas a mantener o recuperar su salud, hacer frente a sus discapacidades o a su muerte" (Leininger, 1999). Los beneficios que brindan los cuidados culturales se orientan al sujeto que tiene una necesidad de ayuda en algún momento de la vida. Para tal efecto, el profesional de enfermería realiza un análisis integral del individuo, despojando el padecimiento y los aspectos socioculturales y religiosos (Castillo, 2008). Visto, como emergen los cuidados de enfermería desde la evidencia, se beneficia a la disciplina enfermera, al concebir la oportunidad de brindar cuidados más contextualizados y por ende significativos.

Los resultados del estudio "primera infancia pumë: ritos de paso, interculturalidad y educación. Una mirada desde la etnografía" muestran que las prácticas de cuidado tienen su principal fundamentación en las prácticas de cuidado tradicional basadas en la sabiduría del chaman, figura respetable, conocedoras de las hierbas y pócimas, lo que lo coloca como un individuo con reconocimiento social, pues es capaz de recuperar la salud de muchos de los suyos. Figura que con su facultad de comunicarse con las fuerzas superiores, está en la capacidad de sembrar el enfermo el elemento psicológico de confianza para su curación. También va a convertirse en el guía espiritual que indicará a los suyos como aprender a prevenirlas. Este aprendizaje de la salud guiado por el chamán, se focaliza adicionalmente al conocimiento elemental de lo que debe comerse o no, a aprender a reconocer lo que es peligroso para la vida. Un conocimiento centrado y apoyado en una actitud disciplinada y organizada para la vida, completada con una formación moral, orientada en última instancia en los consejos de ancianos. En base a los planteamientos descubrimos cual es el valor que le concede la comunidad de búsqueda dentro de sus propios recursos, los modelos para un adecuado cuidado de su salud (García Piña et al, 2015).Es por condiciones como la anterior, que la OMS, propuso en el año 2013 una estrategia global para asegurar el acceso a la Medicina Tradicional Complementaria (MTC) y para promover la investigación, el desarrollo y la innovación con la finalidad de proteger los derechos de propiedad intelectual de los pueblos indígenas y las comunidades locales, así 
Revista científica de la Asociación de Historia y Antropología de los Cuidados (Universidad de Alicante)

como de sus tradiciones de cuidados de salud.

La enfermería transcultural es la única forma de descubrir los mismos profesionales la posibilidad de explorar, entender y aprehender la base cultural de las personas, su argumento y problemáticas sociales, además le reconoce circunscribir su visión personal respecto de la salud y la asistencia. La práctica de enfermería si considera el enfoque transcultural, podrá respetar la diversidad de la sociedad, lo que se ha convertido en una competencia que debe poseer enfermería actual.

\section{MÉTODO}

Para precisar la realidad, se abordó desde el equipaje etnográfico focalizado como metodología analítica explorando la interculturalidad, salud, prevención en la primera infancia Pume, en el periodo de Agosto del 2013- Agosto 2015 durante permanencia intermitente de 30 a 60 días durante esos años, usando la metodología de James Spradley como base epistémica para los dominios y taxonomías, teniendo en cuenta la adecuación teóricoepistemológica fundamentado en el paradigma cualitativo- interpretativo a través del interaccionismo simbólico. Este diseño fue sometido a evaluación de Comité de Ética Científica de la Universidad de Carabobo. El grupo de actores sociales sujeto de estudio estuvo conformado por cinco miembros de la comunidad como informantes claves, y como informantes complementarios cuya selección se realizó sujeta a la saturación teórica. Se eligen una serie de criterios que se consideran necesarios o altamente convenientes para tener una unidad de análisis con las mayores ventajas para los fines que persigue la investigación.

Los rasgos de selección que cumple el grupo de informantes de esta investigación, lo cual puede ser visualizado en esquema presentado a continuación, contentivo de la identificación y características que permitieron su selección. Los datos, como información, imágenes y contenido de entrevistas, se publican con el consentimiento informado de cada una de las personas; documento firmado por los informantes para dar fe de dicha autorización. Los datos de identificación, nombre y apellido, son imaginarios por ética

La técnica que se utilizó para la recolección de la información fue la entrevista focalizada y la observación participante durante la permanencia en el campo de trabajo etnográfico, en la medida que se fueron reuniendo los distintos registros de campo, que conforman el hoy denominado Diario de Ruta, se fue clasificando y organizando en las Notas de Campo, Fichas Descriptivas, Fichas de Registro, Registros Fotográficos y Fonográfico para el análisis primario de lo recolectado, acción que facilitó tres 
elementos claves para el proceso

investigativo; hacer del abordaje un proceso dialéctico, familiarizarme con la información e ir preparando paulatinamente el terreno para un posterior análisis intensivo. Martinez \& Taylor y Bodgan \& Staruss, Corbin \& Glaser, B \& Strauss Goetz, J. \& LeCompte

La construcción del análisis fue enriquecido por los testimonios recogido a través de las entrevistas enfocadas a través del concepto de Análisis Etnográfico de James Spradley, el cual considera un dominio como una categoría. Para la aplicación de este análisis, se consideró los dos tipos de relación semántica de James Spradley: Relaciones Universales y las que fueron expresadas por las informantes. En esta investigación se identifican las: Medio Fin, Razón y Causa - Efecto. La construcción del análisis se realizó a través de los dominios culturales.

\section{RESULTADOS Y SU DISCUSIÓN}

\section{Antropología y interculturalidad en la primera infancia:}

La Primera Infancia Pumé, es el periodo de la niñez que va desde 0 días de nacido hasta cumplido los 5 años de edad, esta etapa es la más importante y significativa en la formación del ser humano, en la cual el niño y la niña mediante su desarrollo y crecimiento van recopilando experiencias y siguiendo ejemplos que las personas jóvenes o adultas les brindan, por eso es fundamental que cada uno de ellos crezca y se forme en lugares sanos con una ayuda incondicional de la familia, la escuela y la comunidad, y así se desenvuelvan en el mundo actual, de una manera adecuada para que en un futuro demuestren autonomía en sus actos, de tal manera se debe tener en cuenta que todo lo que rodea al niño y la niña debe obtener como primordial factor la armonía en todo acto que sea evidenciado en los diferentes contextos en donde hay una socialización, comunicación y cuidado permanente.

La primera infancia Pumé, se encuentran en un pueblo indígena Apureño en Venezuela, conocido coloquialmente como "Yaruro", aunque ésta no es su denominación propia, está ubicado en la zona suroeste del país, en áreas limítrofes con Colombia, y exhibe rasgos culturales característicos de otros pueblos indígenas llaneros vecinos los Hiwi y los subgrupos Hiwi-Cuiva-Chiricoa, Pepo-

Hiwi Amorúa.

Para el conocimiento de la interculturalidad, desde la antropología, fundamentando el cuidado de enfermería, se presenta el enfoque basado en los ritos de paso como claves de respeto hacia esta etnia. A partir de la observación etnográfica con entrevista traducida desde el Pumé al español realizada a los padres de una familia con cuatros niños en edad de primera infancia, los principales Ritos de Paso están en las etapas del crecimiento y desarrollo social de los niños Pumé, son las siguientes:

- Una primera etapa, desde el nacimiento hasta el año y medio o 
Revista científica de la Asociación de Historia y Antropología de los Cuidados (Universidad de Alicante)

dos años, durante la cual el niño está casi todo el tiempo junto a la madre, quien lo cuida y lo amamanta.

- En una segunda etapa, más o menos desde el año y medio o dos años hasta los cuatro, los niños comienzan a explorar el ambiente alrededor de la casa, permaneciendo bajo el cuidado principal de la madre y de los hermanos.

- A partir de los tres años los niños duermen separados de los padres, en un chinchorro propio, y el padre les dedica más tiempo, sobre todo para contarles historias tradicionales. En esta etapa los niños y las niñas comienzan a jugar cada uno por su cuenta.

- De los cuatro y hasta los seis o siete años los niños y las niñas se integran progresivamente al trabajo de los padres, siendo vivido en gran parte esta actividad como juego. Cuando existe, frecuentan la escuela criolla.

- De los siete u ocho años en adelante, y hasta los 12 , se asocian cada vez más a los trabajos de los padres, las niñas en las tareas domésticas y trabajo del conuco y los niños en las actividades de pesca y caza.

- De los doce o trece años en adelante los niños son considerados adultos. Para la niña la etapa está marcada por la primera menstruación, que a veces puede darse antes de la edad indicada, mientras que para los niños este periodo se caracteriza por la adquisición de autonomía en el trabajo y el cambio de la voz.

Las diferentes etapas intermedias no están particularmente marcadas, a excepción del pasaje desde la primera infancia a la segunda cuando, hacia los tres años, se le retiran los collares protectivos que se les habían puesto al nacer. En general, los elementos que permiten a los adultos definir el crecimiento y marcar las diferentes etapas son los siguientes: llanto y primera dentición, gateos y caminar solos, hablar.

El primer rito de paso importante en la familia Pumé, lo constituye el colocar el nombre al niño, la atribución del nombre se realiza en el ámbito familiar, siendo escogido por los padres o los abuelos. Desde hace algunas generaciones, los nombres y los apellidos son de origen criollo, pero se encuentran también apellidos indígenas, derivados de nombres clánicos relacionados con algunos animales como, por ejemplo, el caimán que protegería a los integrantes del grupo familiar que lo lleva y atacaría a sus enemigos. La primera etapa del niño, a través de un rito de paso, caracterizada por el llanto, tanto que es considerado un signo del carácter futuro, lo mismo que la capacidad de agarrar objetos, que suele considerarse una actividad que marca el crecimiento y un indicador de predisposición: agarrar un lápiz de la manera apropiada, por ejemplo, es visto como un signo de predisposición a los estudios; de la misma manera, si juega con casitas, en la edad adulta será muy bueno construyendo los techos de palma y las paredes de barro de las casas.

A $\operatorname{los} 5$ a 6 meses, la dentición es considerada problemática. Aunque no se indican tratamientos particulares para el 
cuidado de la boca, la dentición es un momento importante en la categorización de los ritos de paso, es una etapa de crecimiento de los niños, particularmente por el hecho de que coincide con el progresivo destete y el cambio de la dieta. Por esto, en relación con algunos mitos (el de Bakatsolowa, la sirena, mitad humana mitad pez), al comenzar esta etapa, el chamán o algún anciano de la comunidad le reza el pescado a los niños para protegerlos de cualquier daño que su ingestión pudiera acarrearle en futuro. El ritual se realiza de noche y puede ocupar algunas horas, ya que se trata de hacer referencia a todos los pescados que integran la dieta de los Pumé. No se hace durante el día porque no es posible la concentración necesaria y el chamán puede equivocarse. De hecho, si el rezo no se hace bien, esto podría desencadenar una diarrea $\mathrm{O}$ "gastroenteritis" en el niño. Así, se les reza el pescado con rigurosidad al niño y a la niña con 3 o 4 meses. A la niña se le vuelve a hacer el rezo durante la primera menstruación, cuando también se le rezan las manos, para que sea más hábil y diestra en la elaboración del casabe.

Por lo que se refiere a los gateos, parece haber consenso en que los niños y las niñas, sin particulares diferencias, comienzan a gatear entre los cinco y los seis meses; si estos comienzan más tarde, por ejemplo a los ocho y nueve meses, se considera un retraso. Los gateos se realizan en el espacio doméstico interno, bajo la mirada de la madre o de algún otro familiar. Las enfermedades a veces atrasan el gateo y el caminar, lo mismo que algunos comportamientos como, por ejemplo, que el padre del niño mate una culebra de agua, que es dañino porque ablanda las piernas del niño. A partir del noveno mes el niño comienza a levantarse, sólo o con ayuda de los familiares, hasta que, al cumplir más o menos el primer año de vida, lo consigue y comienza a deambular.

Aunque es la madre la indicada como la figura fundamental de este aprendizaje, el padre no está ausente. De hecho los mayores detalles son declarados por ellos, como las referencias de que los niños se ayudan con sillas y otros elementos para levantarse. Los niños, una vez que han aprendido a levantarse, corretean por toda la casa, siguiendo a los adultos, quienes tienen un papel activo en la enseñanza de los primeros pasos. Contemporáneamente a estos desarrollos, los niños comienzan a hablar, desde los primeros balbuceos hasta la formulación de frases enteras. Es afirmación común que los balbuceos de los niños no tienen sentido, mientras que las primeras palabras parecen referirse particularmente a los nombres de los padres y a la comida, bajo inducción de los adultos, quienes repiten los nombres de papá y mamá en Pumé tocándose la cabeza y señalándose a sí mismos. Aunque es la madre quien habla más a menudo a los niños en el dialecto Pumé, lo mismo hace el padre, el que mezcla con más facilidad este dialecto con el castellano. 
Todos estos procesos se realizan durante la primera etapa del crecimiento del niño y de la niña. A partir del aprendizaje de las diferentes habilidades citadas, los niños entran en otra etapa, cuando amplían su espacio de vivencia, saliendo de la casa solos o juntándose a los otros niños de la comunidad. En este período, la actitud de los padres cambia en relación con la mayor autonomía alcanzada por los niños, mientras que la familia más amplia asume mayores funciones. Estos cambios en la vida del niño Pumé están periódicamente marcados por algún evento especial, como puede ser el cambio de los collares y pulseras protectoras o, en el caso de la niña, la perforación de las orejas para ponerles aretes o zarcillos, a la manera occidental.

Cuando el niño asume la categoría de tsikirrinü̈̈yo, puede darse una fiesta en el ámbito familiar, lo que parece ser un residuo de alguna ceremonia tradicional relacionada con el cambio de los collares y la nueva protección que los niños necesitan. De alguna manera, después de la acción de evangelización de las diferentes iglesias cristianas en la región, una parte de estos rituales ha sido substituida por el bautizo cristiano que sirve para remarcar y celebrar el crecimiento de los niños, protegerlos mejor y adquirir nuevas relaciones o nuevos lazos con individuos de la misma comunidad o de afuera a través del compadrazgo. De esta manera se crean afiliaciones nuevas, por ejemplo comadre y compadre, por influencia de la sociedad occidental.

No hay actualmente rituales especiales codificados entre la infancia y la pubertad, aunque el ingreso a la escuela criolla puede ser considerado como un rito de paso o momento especial para los niños, ya que marca de manera profunda el tránsito del espacio familiar a otro comunitario, definido por nuevas relaciones de poder y acceso a contenidos no pertenecientes a la cultura local. Por otro lado, en el caso de la niña, el fin de la infancia está fuertemente marcado por la menstruación que, como veremos, conlleva rituales especiales; mientras que, en el caso de los niños, se trata de un proceso no particularmente remarcado y el mismo cambio de nombre es un evento intrafamiliar. Generalmente, los mismos familiares añaden un apodo basado en las características del niño

A modelo de ejemplo se deja como las ciencias de la enfermería, de raíces esencialmente desde la antropología, como se lograría la necesaria simbiosis entre la práctica de enfermería y los cuidados culturales. a continuación, fragmentos desde la metodología utilizada según James Spradley.

TAXONOMÍA 2: "EL JUEGO POTENCIA EL DESARROLLO Y EL APRENDIZAJE EN LAS NIÑAS Y LOS NIÑOS PUME" 
Cultura de los Cuidados

\begin{tabular}{|c|c|c|}
\hline Placentero & $\begin{array}{l}\text { El impulso } \\
\text { natural de las } \\
\text { niñas y los niños } \\
\text { hacia el juego }\end{array}$ & \begin{tabular}{ll|} 
Pasa de & un \\
juego & \\
solitario & $\mathrm{a}$ \\
juegos & \\
grupales &
\end{tabular} \\
\hline $\begin{array}{l}\text { Movimiento } \\
\text { del cuerpo }\end{array}$ & $\begin{array}{l}\text { Logro de } \\
\text { maduración } \\
\text { motora gruesa y } \\
\text { fina }\end{array}$ & $\begin{array}{l}\text { Equilibrio, } \\
\text { subir y bajar } \\
\text { árboles, } \\
\text { agarre del } \\
\text { lápiz, } \\
\text { recortar } \\
\text { figuras. }\end{array}$ \\
\hline Organización & $\begin{array}{lr}\text { Forma } & \text { de } \\
\text { aprendizaje } & y \\
\text { sentido a } & \text { las } \\
\text { actividades } & \end{array}$ & $\begin{array}{l}\text { Integra los } \\
\text { juegos } \\
\text { populares en } \\
\text { su familia a } \\
\text { la escuela }\end{array}$ \\
\hline Focalización & $\begin{array}{l}\text { Una forma de } \\
\text { concentración }\end{array}$ & $\begin{array}{l}\text { Integra a los } \\
\text { juegos, los } \\
\text { rituales, } \\
\text { mitos, juegos } \\
\text { prohibidos }\end{array}$ \\
\hline $\begin{array}{l}\text { Dominio } \\
\text { sí mismo }\end{array}$ & $\begin{array}{l}\text { Expresa sus } \\
\text { cualidades en la } \\
\text { interrelación con } \\
\text { los demás }\end{array}$ & $\begin{array}{lr}\text { Aprende r a } \\
\text { compartir los } \\
\text { tallados de } \\
\text { madera, } \\
\text { collares. }\end{array}$ \\
\hline Respeto & $\begin{array}{l}\text { Colabora } \\
\text { espontáneament } \\
\text { e en diferentes } \\
\text { actividades de } \\
\text { juegos }\end{array}$ & $\begin{array}{l}\text { Respeta } \\
\text { sobre los } \\
\text { ancianos, y } \\
\text { respeto a los } \\
\text { niños y niñas }\end{array}$ \\
\hline Solidaridad & $\begin{array}{l}\text { Toma en cuenta } \\
\text { la idea de los } \\
\text { demás }\end{array}$ & \begin{tabular}{lr}
\multicolumn{2}{l}{ Observa } \\
entiende \\
como puede \\
una niña \\
Pumé
\end{tabular} \\
\hline Atención & $\begin{array}{l}\text { Expresa temores, } \\
\text { inquietudes y } \\
\text { curiosidad ante } \\
\text { algunas } \\
\text { situaciones, } \\
\text { manifiesta a } \\
\text { través del juego }\end{array}$ & $\begin{array}{l}\text { Le concede } \\
\text { importancia } \\
\text { a los juegos } \\
\text { prohibidos, } \\
\text { leyendas y } \\
\text { cuentos }\end{array}$ \\
\hline $\begin{array}{l}\text { Respeto por } \\
\text { los demás }\end{array}$ & $\begin{array}{l}\text { Elige materiales } \\
\text { de juego que más } \\
\text { le agrada }\end{array}$ & $\begin{array}{lr}\text { Sabe } & \text { de } \\
\text { pintura } & y \\
\text { significado } \\
\text { en el cuerpo } \\
\text { descubre re la }\end{array}$ \\
\hline
\end{tabular}

\begin{tabular}{|c|c|c|}
\hline & & $\begin{array}{ll}\text { tempera } & \mathrm{y} \\
\text { acuarela } & \end{array}$ \\
\hline Seguridad & 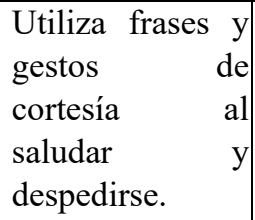 & $\begin{array}{l}\text { Aprende a } \\
\text { saludar en } \\
\text { lengua } \\
\text { castellana } \\
\text { timidamente. }\end{array}$ \\
\hline Cooperación & $\begin{array}{l}\text { Participa en el } \\
\text { establecimiento } \\
\text { y cumplimiento } \\
\text { de normas de } \\
\text { juego. }\end{array}$ & \begin{tabular}{|lr} 
Sigue ras \\
normas de la \\
maestra & sin \\
saltarse & \\
ninguna &
\end{tabular} \\
\hline $\begin{array}{l}\text { Manejo con } \\
\text { cierta } \\
\text { destreza } \\
\text { algunos } \\
\text { objetos e } \\
\text { instrumentos }\end{array}$ & $\begin{array}{l}\text { Constituye uno } \\
\text { de los alcances } \\
\text { más significativo } \\
\text { en aula }\end{array}$ & $\begin{array}{l}\text { Recolecta } \\
\text { taparas, } \\
\text { elaboración } \\
\text { de collares, } \\
\text { tallado de } \\
\text { madera }\end{array}$ \\
\hline
\end{tabular}

Fuente: elaboracion propia

\section{DOMINIO 3: LA EXPRESIÓN CORPORAL COMO RITO DE PASO EN EL CONTEXTO EDUCATIVO A TRAVÉS DE LA EXPRESIÓN ARTÍSTICA}

\section{Relación Semántica: Medio...Fin}

Ejemplo: Como parte de sus experiencias más tempranas, las niñas y los niños utilizan la dramatización, el teatro,(narrativa, cuentos, imaginación) integrando el uso de formas y líneas a través de estrategias pedagógicas empleadas en el aula: el dibujo libre en creyones y tempera.

\begin{tabular}{|l|l|l|}
\hline \multicolumn{1}{|c|}{$\begin{array}{c}\text { Términos } \\
\text { incluidos }\end{array}$} & $\begin{array}{l}\text { Relación } \\
\text { Semántica }\end{array}$ & Dominio \\
\hline $\begin{array}{l}\text { Mediante el } \\
\text { empleo re de } \\
\text { dibujos libres, } \\
\text { colores, recortes } \\
\text { de material de } \\
\text { desecho, uso de } \\
\text { semillas, y } \\
\text { diseño de de } \\
\text { collares. }\end{array}$ & $\begin{array}{l}\text { Se } \\
\text { fanorecen y y }\end{array}$ & $\begin{array}{l}\text { Realiza la } \\
\text { expresión } \\
\text { corporal } \\
\text { como } \\
\text { apreciación } \\
\text { artística } \\
\text { usando la } \\
\text { utilización }\end{array}$ \\
\hline
\end{tabular}


Revista científica de la Asociación de Historia y Antropología de los Cuidados (Universidad de Alicante)

\begin{tabular}{|c|c|}
\hline $\begin{array}{l}\text { Expresa } \\
\text { mediante el } \\
\text { lenguaje oral: } \\
\text { palabras en } \\
\text { Pumé algunos } \\
\text { elementos } \\
\text { narrativos } \\
\text { ejemplo: la bola } \\
\text { de fuego. } \\
\text { Expresa } \\
\text { mediante } \\
\text { lenguaje } \\
\text { Gestual: alegría, } \\
\text { miedo, timidez, } \\
\text { Expresa } \\
\text { mediante } \\
\text { lenguaje } \\
\text { corporal, el uso } \\
\text { del ritmo a } \\
\text { través de la } \\
\text { música: } \\
\text { Realiza } \\
\text { representaciones } \\
\text { reales de cómo } \\
\text { es la danza } \\
\text { Realiza } \\
\text { representaciones } \\
\text { imaginarias } \\
\text { teatrales } \\
\text { sencillas, uso de } \\
\text { materiales } \\
\text { ornamentales y } \\
\text { alfarería pumé }\end{array}$ & \begin{tabular}{|l} 
de collares, \\
empleo y \\
dominio de \\
la maraca y \\
tambor.
\end{tabular} \\
\hline
\end{tabular}

Fuente: elaboracion propia

\section{La necesaria simbiosis entre la enfermería transcultural en una comunidad indígena}

En la comunidad Pumé las prácticas de cuidado sientan sus bases en rituales de curación y religiosos donde la fe en un ser superior cumple un papel muy importante para el afrontamiento de los procesos de salud-enfermedad y muerte. Lo anterior, se ha considerado parte del cuidado al que ya en diferentes contextos han hecho alusión distintos autores (Navas \& Villegas, 2006).
A continuación, se presentan siete estrategias que, conforme a las necesidades de la primera infancia Pumë, pueden retomarse para la práctica cotidiana de cuidar considerando los ritos de paso como claves de respeto en las clínicas de crecimiento y desarrollo en el cuidado de enfermería desde el área preventivo:

1.-Los ritos de paso, son el instrumento principal utilizado para restablecer el equilibrio y, de allí, su importancia en la reproducción y producción cultural. Todas las comunidades Pumé observados realizan ritos durante los nacimientos, así como en los momentos de transición de los niños a la vida adulta.

2.-Se puede categorizar estos rituales en diferentes tipos a partir del particular fenómeno que regulan $\mathrm{y}$, sobre todo, de su desarrollo: ritos de separación, como es el caso de los funerales, de incorporación, como en los matrimonios, y de transición, como en el caso de los ritos de iniciación. Sin embargo, particularmente en el caso de las etapas que marcan el desarrollo físico y la evolución social de la primera infancia, podemos encontrar aspectos de todos los tres tipos, como se ha señalado en los dominios culturales.

3.-Es importante resaltar que los casos tomados en consideración en la presente investigación indican que existe una variabilidad marcada de los tiempos de cambio, confirmando que las fases de 
crecimiento están definidas por el universo cultural local más que por los eventos biológicos.

4.-La existencia de rituales que marcan el pasaje de un estadio al otro de la vida de los niños y de las niñas Pumé impone el análisis de las etapas con la finalidad de hacerla aprehensible a la conciencia de los actores sociales. Aunque hay una evidente correspondencia entre desarrollo psicológico y cognitivo de los niños y etapas del desarrollo, la información aportada por la etnografía, apuntan hacia la categorización cultural de las mismas, es decir, que los datos recolectados se refieren a lo que los padres, informantes claves, declararon más que a la observación clínica de los niños.

5.-De cualquier manera, en este estudio se mantuvo como referente que las estructuras psicológicas se forman en directa relación con la maduración biológica del niño, pero no necesariamente en concordancia con la atribución de lugar social, variando este en relación a cada cultura.

6.-Las sociedades indígenas Pumé, la represión de las emociones marca la formación de la personalidad de la primera infancia Pume, mientras que en otras el control es más blando, dejando un mayor margen de libertad al desarrollo personal de las emociones. Parece valer aquí una diferenciación interesante entre los controles impuestos a los niños de los impuestos a las niñas, siendo sobre éstas que recae el mayor

peso de la represión familiar.

7.-Finalmente, es necesario hacer referencia también a los modelos de desarrollo psicológico que enunciamos en las precisiones teóricas, Ya observamos que la etnografía nos permite averiguar completamente la validez del modelo Piagetiano en relación a las etapas de desarrollo que se ha identificado. Para esto sería necesario elaborar una serie de pruebas y una observación psicológica determinada por protocolos intra-culturales. Sin embargo, algo sí podemos inferir de los datos recolectados: la fase sensorio motor, que según Jean Piaget se completa a los dos años de edad, parece realizarse más o menos en ese rango temporal. Sin embargo, en el caso de la llamada fase pre-operacional, que se cumpliría entre los dos y siete años, tenemos la impresión que, en el caso de los niños y niñas indígenas Pumé, esta se cumple en un tiempo menor, quedándose completada hacia los cinco o seis años de edad. De hecho, si lo que caracterizaría esta fase es la relación con el mundo extra-familiar y la estructuración de las relaciones sociales, con distinción consiente entre parientes y no pariente, no cabe duda que los niños y niñas indígenas Pumé alcanzan tempranamente este estadio de desarrollo.

\section{CONCLUSIONES}

La enfermería, como parte de un sistema de salud, también tendría que promover el cumplimiento de esta estrategia 
Revista científica de la Asociación de Historia y Antropología de los Cuidados (Universidad de Alicante)

mediante el respeto y conocimiento de los tipos de cuidado a la salud y a la enfermedad que se generan en los diferentes grupos sociales y que permiten conservar la cultura, pero aún más importante, obedecen al mantenimiento del bienestar y la vida. En la primera infancia Pume, es de vital importancia, la interpretación de los ritos de paso, son el instrumento principal utilizado para restablecer el equilibrio y, de allí, su importancia en la reproducción y producción cultural. Todas las comunidades Pumé observados realizan ritos durante los nacimientos, así como en los momentos de transición de los niños a la vida adulta. Resulta conveniente que se haga una reflexión hacia los modelos de cuidado utilizados, que están descontextualizados de la cultura en Venezuela y Latinoamérica, y comenzar a crear alternativas de cuidado que surjan de quien los recibe y no de quien los otorga.

La ciencia de enfermería, debe emerger para que se dé en la práctica ese respeto reclamado hacia todos los niños con diferentes culturas que integran la comunidad plural del siglo XXI, es preciso comprender que es imposible respetar lo que no se conoce. Dicho de otro modo, para ejercer eficazmente la enfermería transcultural se debe profundizar en el conocimiento de esa comunidad plural., especialmente las etnias indígenas. Y aún más, no sólo existe una enfermería transcultural, sino que más bien, este tipo de enfermería holística y armonizadora es el ideal u objetivo a alcanzar en una sociedad plural. La necesaria reflexión desde la antropología en el cuidado de enfermería transcultural, en este momento debe hacerse en esta mirada, los cuidados culturales en la Primera infancia, comprenderán actividades orientadas a preservar o mantener, adaptar o negociar, reorientar o reestructurar los cuidados otorgados a los niños. El Sol Naciente, de Madeleine Leninger, representa el universo donde está inserta la persona, el que debe ser considerado por el profesional de enfermería, con una mirada holística de diversidad cultural para brindar cuidados seguros, responsables y congruentes con la cultura del otro, que es propia de su contexto original.

La cultura y el cuidado acompañan al ser humano desde que nace y van siendo compartidas y transferidas de una generación a otra, utilizándose como herramientas para preservar la existencia humana. La salud y los cuidados son universales, pero las formas de cómo estamos realizando esos cuidados influye en la forma de concebir la salud definidas de manera diferente según las culturas, esto refleja la diversidad. Es por ello que en el contexto multicultural en el que vivimos, toma importancia incorporar las creencias y valores de las personas y de esta forma proporcionar cuidados de enfermería efectivos, satisfactorios y coherentes con su cultura. 


\section{BIBLIOGRAFÍA}

Barona de Infante, N., \& Álvarez, L. (1986). El proceso de salud-enfermedad: un fenómeno social. Investigación y Educación en Enfermería, 4(2), 47-54.

Cánovas Tomás, M. A. (2008). La relación de ayuda en enfermería. Una lectura antropológica sobre la competencia relacional en el ejercicio de la profesión. Tesis de doctorado. Murcia: Universidad de Murcia. Recuperado de http://bit.ly/21Iy873

Cardoso, M. A., Pascual, R., Moreno, G. M., Figueroa, M. E., \& Serrano, C. (2007). Investigación cualitativa y fenomenología en salud. Vertientes Revista Española en Ciencias de la salud, 10(1-2), 25-32. Recuperado de http://bit.ly/2koaE6S

Castillo, J. A. (2008). El cuidado cultural de enfermería. Necesidad y relevancia. Revista Habanera de Ciencias Médicas, 7(3), 1-7. Recuperado de http://bit.ly/2ocmF8

García Piña, E. V. (2015). Prácticas de cuidado tradicional y espiritual en una comunidad indígena nahua de Zongolica, Veracruz. Tesis inédita de maestría. México: Universidad Nacional Autónoma de México.

García, P. E. V., Cardoso, G. M. A., Serrano, S. C., \& Ostiguín, M. R. M. (2015). Prácticas de cuidado tradicional y espiritual en una comunidad indígena nahua. Cultura de los cuidados, 19 (41), 34-43. doi: http://dx.doi. org/10.14198/cuid.2015.41.06

Hernández, L., \& Vásquez, M. L. (2010). Practices and beliefs about exclusive breastfeeding by women living in Commune 5 in Cali, Colombia. Colombia Médica, 41 (2), 161-170. Recuperado de http://bit.ly/2166xJH

Leininger, M. (1999). Cuidar a los que son de culturas diferentes requiere el conocimiento y las aptitudes de la enfermería transcultural. Cultura de los cuidados, 3(6), 5-12. doi: http://dx.doi.org/10.14198/cuid.1999.6.01

Martínez, M. (2006). Ciencia y arte en la metodología cualitativa. $2^{\mathrm{a}}$ ed. México: Trillas.

Navas, C., \& Villegas, H. (2006). Espiritualidad y salud. Ciencias de la educación, 27 (1), 35-38. Recuperado de: $\mathrm{http} / / \mathrm{bit} .1 \mathrm{ly} / 2 \mathrm{IIcXSu}$

Estrategia de la OMS sobre medicina tradicional 2014- 2023, Organización Mundial de la Salud, 2013. Ginebra: OMS. Recuperado de http://bit.ly/1Q3GLA5

Pérez, S. (2009). Enfermería transcultural como método para la gestión del cuidado en una comunidad urbana. Revista Cubana de Enfermería, 25 (3-4), 1-8. Recuperado de http://bit.ly/1ppnhXq

Quilez Fajardo, L., \& Ferrer Ferrándiz, E. (2015). Cuando la enfermería deviene teología. Cultura de los Cuidados, 19, 41, 23-33. doi: http://dx.doi.org/10.14198/ cuid.2015.41.05

Ramos Lanfot, C. (2011). Prácticas culturales de cuidado de gestantes indígenas que viven en el Resguardo Zenú ubicado en la Sabana de Córdoba. Tesis de maestríaBogota: Universidad Nacional de Colombia. Recuperado de http://bit.ly/21NwWMv

Sánchez Herrera, B. (2004). La dimensión espiritual del cuidado de enfermería en situaciones de cronicidad y muerte. Aquichán, 4, 6-9.

Ritzer, G., Requena, D., \& Revenga, R. (2001).Teoría sociológica clásica. Madrid: McGraw-Hill, 2001. 
Revista científica de la Asociación de Historia y Antropología de los Cuidados (Universidad de Alicante)

Spradley J. (1979). The Ethnographic procedimientos para desarrollar la teoría Interview. New York: Harcourt B.J.C. fundamentada, Trad.). Colombia: Publishers.

Zimmerman.

Siles-González， J. (2016). La utilidad práctica de la Epistemología en la clarificación de la pertinencia teórica y metodológica en la disciplina enfermera, Index de Enfermería, 25(1-2), 86-92.

Staruss, A., y Corbin, J. (2000). Bases de la investigación cualitativa. Técnicas y
Sánchez, F. (2000). Prontuario de investigación documental $y$ de campo. México: Trillas.

Taylor, S.J., \& Bodgan, R. (1984). Introducción a los métodos cualitativos de la investigación. Buenos Aires: Paidos.

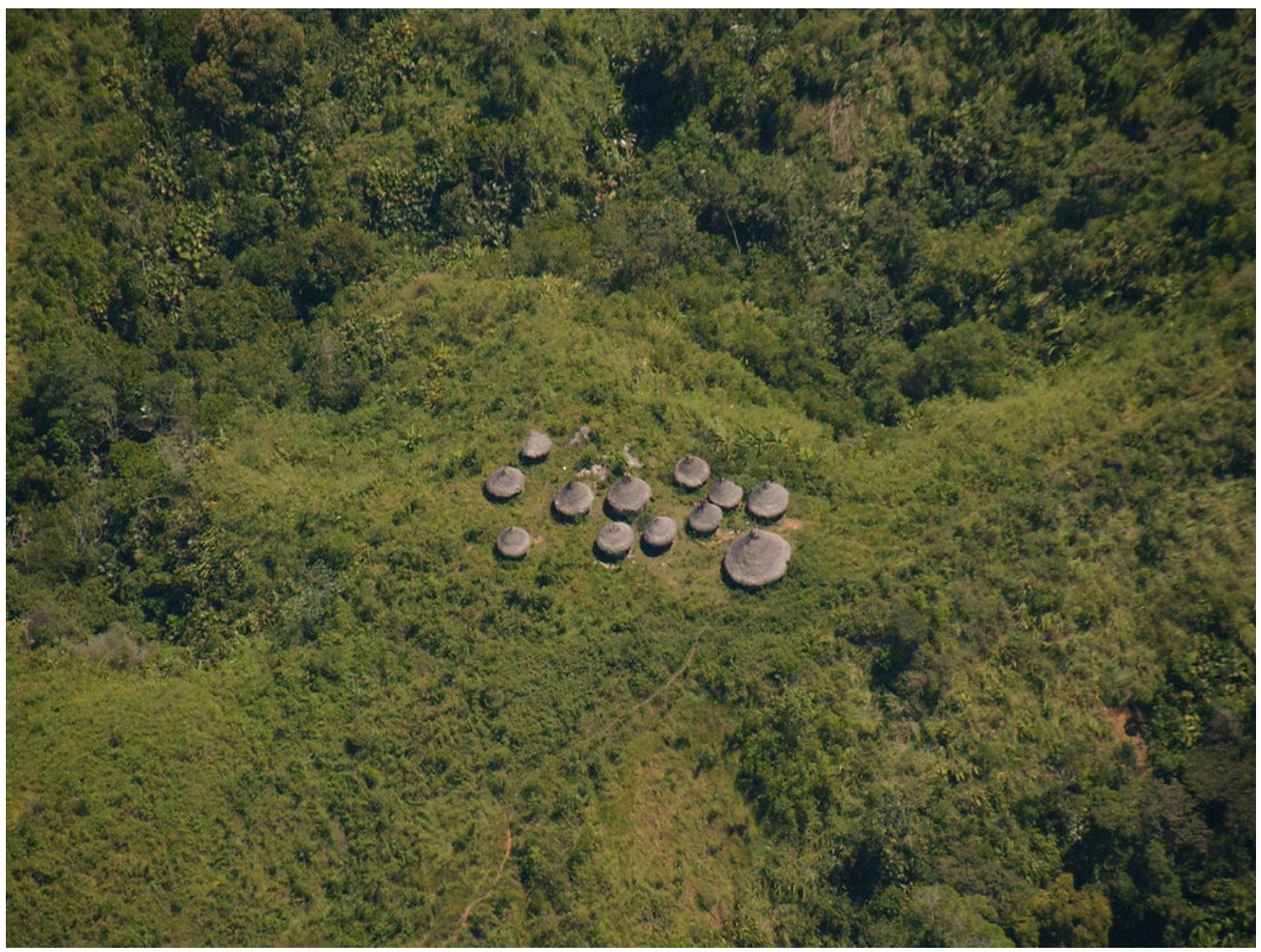

Fuente: by maohill. CC BY 2.0 license. https://search.creativecommons.org/photos/1463a235abb4-4681-a98a-8f08ac88fac7 